\title{
Weighted evolving networks: coupling topology and weights dynamics
}

\author{
Alain Barrat, ${ }^{1}$ Marc Barthélemy, ${ }^{2}$ and Alessandro Vespignani ${ }^{1}$ \\ ${ }^{1}$ Laboratoire de Physique Théorique (UMR du CNRS 8627), \\ Batiment 210, Université de Paris-Sud 91405 Orsay, France \\ ${ }^{2}$ CEA-Centre d'Etudes de Bruyères-le-Châtel, Département de Physique \\ Théorique et Appliquée BP12, 91680 Bruyères-Le-Châtel, France
}

\begin{abstract}
We propose a model for the growth of weighted networks that couples the establishment of new edges and vertices and the weights' dynamical evolution. The model is based on a simple weightdriven dynamics and generates networks exhibiting the statistical properties observed in several real-world systems. In particular, the model yields a non-trivial time evolution of vertices' properties and scale-free behavior for the weight, strength and degree distributions.

PACS numbers: 89.75.-k, -87.23.Ge, 05.40.-a
\end{abstract}

The last years have witnessed a hectic activity devoted to the characterization and understanding of networked structures as diverse as ecological and biological systems or the Internet and the WWW 1, 2, 3, 4]. These networks generally exhibit complex topological properties such as the small-world phenomenon [5] and scale-free behavior [1]. The need for explaining these complex topological features has led to a new modeling framework that focuses on the dynamical evolution and growth of networks [6]. In this perspective, a wide array of models aimed at capturing various properties of real networks have been formulated [3]. These models, however, do generally consider only the topological structure and do not take into account the interaction strength - the weight of the link- that characterizes real networks. For example, the diversity of the predator-prey interaction and of metabolic reactions is considered a critical ingredient of ecosystems [7, 8] and metabolic networks [9], respectively; in social systems, the weight of interactions is very important in the characterization of the corresponding networks [10]. Similarly, the Internet traffic [4] or the number of passengers in the airline network [2, 11, 12] are crucial quantities in the study of these systems. Interestingly, recent studies $[9,12,13,14]$ have shown that weighted networks exhibit additional complex properties such as broad distributions and non-trivial correlations of weights that do not find an explanation just in terms of the underlying topological structure.

In this Letter, we define a simple model for weighted networks that considers the basic evolution of the system as driven by the weight properties of edges and vertices. In addition, differently from early models proposed in the past [15, 16], we allow the dynamical evolution of weights during the growth of the system. This mimics the evolution and reinforcements of interactions in natural and infrastructure networks. The generated networks display power-law behavior for the weight, degree and strength distributions with non-trivial exponents depending on the unique parameter defining the model's dynamics. These results suggest that the inclusion of weights in networks modeling naturally explains the diversity of scale-free behavior empirically observed in real networked structures. Strikingly, the weight-driven growth recovers an effective preferential attachment for the topological properties, providing a microscopic explanation for the ubiquitous presence of this mechanism.

Weighted networks are usually described by a matrix $w_{i j}$ specifying the weight on the edge connecting the vertices $i$ and $j$, with $i, j=1, \ldots, N$ where $N$ is the size of the network ( $w_{i j}=0$ if the nodes $i$ and $j$ are not connected). In the following we will consider only the case of symmetric weights $w_{i j}=w_{j i}$. Prototypical examples of weighted networks can be found in the world-wide airport network (WAN) 11, 12 and the scientific collaboration network (SCN) 17, 18]. In the airport network each given weight $w_{i j}$ is the number of available seats on direct flights connections between the airports $i$ and $j$. In the SCN the nodes are identified with authors and the weight depends on the number of co-authored papers [12, 17]. A first topological characterization of networks is obtained with the vertices degree $k$, number of connected neighbors, and the associated probability distribution $P(k)$. Complex networks often exhibits a power-law degree distribution $P(k) \sim k^{-\gamma}$ with $2 \leq \gamma \leq 3$ and measurements of weighted networks confirm this evidence [11, 12]. Similarly, a first characterization of weights is obtained by the distribution $P(w)$ that any given edge has weight $w$. In complex networks such as the WAN and SCN this distribution is heavy tailed and spans several orders of magnitude [12, 13]. Along with the degree of a node, a very significative measure of the network properties in terms of the actual weights is obtained by looking at the vertex strength $s_{i}$ defined as 12,15

$$
s_{i}=\sum_{j \in \mathcal{V}(i)} w_{i j},
$$

where the sum runs over the set $\mathcal{V}(i)$ of neighbors of $i$. The strength of a node integrates the information about its connectivity and the weights of its links, and can be considered as the natural generalization of the connectivity. For instance, in the case of the WAN the strength provides the actual traffic going through the vertex $i$ and 


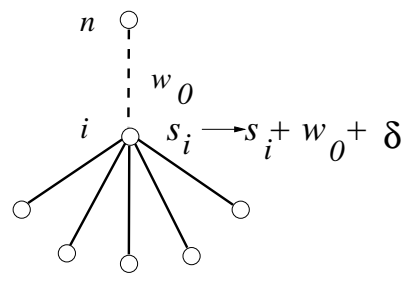

FIG. 1: Illustration of the construction rule. A new node $n$ connects to a node $i$ with probability proportional to $s_{i} / \sum_{j} s_{j}$. The weight of the new edge is $w_{0}$ and the total weight on the existing edges connected to $i$ is modified by an amount equal to $\delta$.

is an obvious measure of the size and importance of each airport. This quantity obviously increases with the vertex degree $k_{i}$ and usually displays the power-law behavior $s \sim k^{\beta}$, with the exponent $\beta$ varying as a function of the specific network. Finally, we note that in most cases, the empirical distribution of $s$ is heavy-tailed [12], analogously to the power-law decay of the degree distribution.

Previous approaches to the modeling of weighted networks focused on growing topologies where weights were assigned statically, i.e. once for ever, with different rules related to the underlying topology [15, 16]. These mechanisms, however, overlook the dynamical evolution of weights according to the topological variations. We can illustrate this point in the case of the airline network. If a new airline connection is created between two airports it will generally provoke a modification of the existing traffic of both airports. In general, it will increase the traffic activity depending on the specific nature of the network and on the local dynamics. In the following we will implement a model that takes into account the coupled evolution in time of topology and weights.

The model dynamics starts from an initial seed of $N_{0}$ vertices connected by links with assigned weight $w_{0}$. At each time step, a new vertex $n$ is added with $m$ edges that are randomly attached to a previously existing vertex $i$ according to the probability distribution

$$
\Pi_{n \rightarrow i}=\frac{s_{i}}{\sum_{j} s_{j}} .
$$

This rule relaxes the usual degree preferential attachment, focusing on a strength driven attachment in which new vertices connect more likely to vertices handling larger weights and which are more central in terms of the strength of interactions. The weight of each new edge is fixed to a value $w_{0}$. Moreover, the presence of the new edge $(n, i)$ will introduce variations of the existing weights across the network. In particular, we consider the local rearrangements of weights between $i$ and its neighbors $j \in \mathcal{V}(i)$ according to the simple rule

$$
w_{i j} \rightarrow w_{i j}+\Delta w_{i j}
$$

where

$$
\Delta w_{i j}=\delta \frac{w_{i j}}{s_{i}}
$$

This rule considers that the establishment of a new edge of weight $w_{0}$ with the vertex $i$ induces a total increase of traffic $\delta$ that is proportionally distributed among the edges departing from the vertex according to their

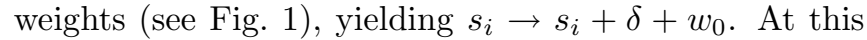
stage, it is worth remarking that while we will focus on the simplest model with $\delta=$ const, different choices of $\Delta w_{i j}$ with heterogeneous $\delta_{i}$ or depending on the specific properties of each vertex $\left(w_{i j}, k_{i}, s_{i}\right)$ can be considered [19]. Finally, after the weights have been updated the growth process is iterated by introducing a new vertex with the corresponding re-arrangement of weights.

The weight driven attachment (Eq. (2) ) appears to be a plausible mechanism in many technological networks. In the Internet new routers connect to more central routers in terms of bandwidth and traffic handling capabilities and in the airport networks new connections are generally established to airports with a large passenger traffic. Even in the SCN this mechanism might play a role since an author with more co-authored papers is more visible and open to further collaborations. Also the weights dynamics can be traced back to simple dynamical processes. Indeed, the quantity $w_{0}$ sets the scale of the weights and without loss of generality we can use the rescaled quantities $w_{i j} / w_{0}, s_{i} / w_{0}$ and $\delta / w_{0}$. For the sake of simplicity we can set $w_{0}=1$ and the model depends only on the dimensionless parameter $\delta$, that is the fraction of weight which is 'induced' by the new edge onto the others. According to the value of $\delta$, different scenarios are possible. If the induced weight is $\delta \approx w_{0}=1$ we mimic situations in which an appreciable fraction of traffic generated by the new connection will be dispatched in the already existing connections. This is plausible in the airport networks where the transit traffic is rather relevant in hubs. In the case of $\delta<1$ we face situations such as the SCN where it is reasonable to consider that the birth of a new collaboration (co-authorship) is not triggering a more intense activity on previous collaborations. Finally, $\delta>1$ is an extreme case in which a new edge generates a sort of multiplicative effect that is bursting the weight or traffic on neighbors.

In the model, the time is measured with respect to the number of vertices added to the graph, resulting in the definition $t=N-N_{0}$, and the natural time scale of the model dynamics is the network size $N$. The network's evolution can be inspected analytically by studying the time evolution of the average value of $s_{i}(t)$ and $k_{i}(t)$ of the $i$-th vertex at time $t$, and by relying on the continuous approximation that treats $k, s$ and the time $t$ as continuous variables [1, 3]. When a new edge is added to the network, the strength $s_{i}$ of vertex $i$ can increase either if the edge connects directly to $i$ or to one of its 


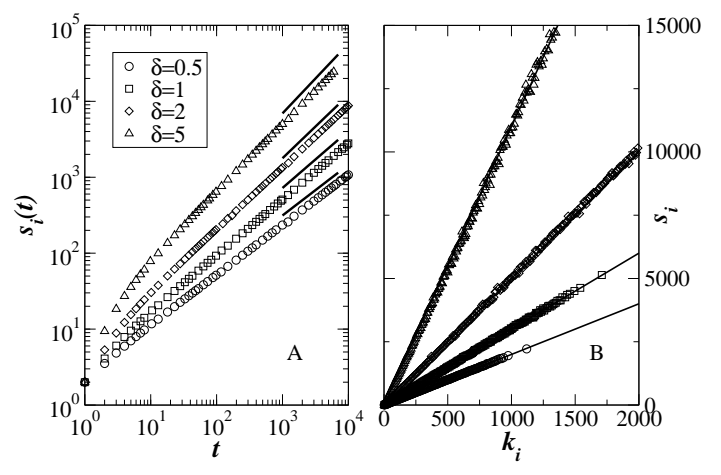

FIG. 2: (A) Evolution of the strength of vertices during the growth of the network, for various values of $\delta$; The thick lines are the predicted power laws $t^{a}, a=(1+2 \delta) /(2+2 \delta)(m=2$, $N=10^{4}$ ). (B) Strength $s_{i}$ versus $k_{i}$ for various values of $\delta$; the straight lines are the predictions $s_{i}=(1+2 \delta) k_{i}$.

neighbor. The evolution equation for $s_{i}$ is thus given by

$$
\begin{aligned}
\frac{d s_{i}}{d t} & =m \frac{s_{i}}{\sum_{j} s_{j}}(1+\delta)+\sum_{j \in \mathcal{V}(i)} m \frac{s_{j}}{\sum_{l} s_{l}} \delta \frac{w_{i j}}{s_{j}} \\
& =\frac{2 \delta+1}{2 \delta+2} \frac{s_{i}(t)}{t},
\end{aligned}
$$

where the last expression is recovered by noticing that each added link increases the total strength by an amount equal to $2+2 \delta$ thus implying that $\sum_{i} s_{i}(t) \approx 2 m(1+\delta) t$. This equation can be readily integrated with the initial condition $s_{i}(t=i)=m$, yielding

$$
s_{i}(t)=m\left(\frac{t}{i}\right)^{\frac{2 \delta+1}{2 \delta+2}}
$$

Similarly, the rate equation for the degree evolution reads

$$
\frac{d k_{i}}{d t}=m \frac{s_{i}(t)}{\sum_{j} s_{j}(t)}=\frac{s_{i}(t)}{2(1+\delta) t}
$$

that implies $k_{i}(t)=s_{i}(t) /(2 \delta+1)$. The proportionality relation $s \sim k$, that implies $\beta=1$, is particularly relevant since it states that the weight-driven dynamics generates in Eq. (2) an effective degree preferential attachment that is parameter independent. This highlights an alternative microscopic mechanism accounting for the presence of the preferential attachment dynamics in growing networks.

The behavior of the various statistical distributions can be computed by noticing that the time at which the node $i$ enters the network is uniformly distributed in $[0, \mathrm{t}]$ and

$$
P(k, t)=\frac{1}{t+N_{0}} \int_{0}^{t} \delta\left(k-k_{i}(t)\right) d i,
$$

where $\delta(x)$ is the Dirac delta function. Solving the above integral and considering the infinite size limit $t \rightarrow \infty$ yields $P(k) \sim k^{-\gamma}$ with

$$
\gamma=\frac{4 \delta+3}{2 \delta+1}
$$
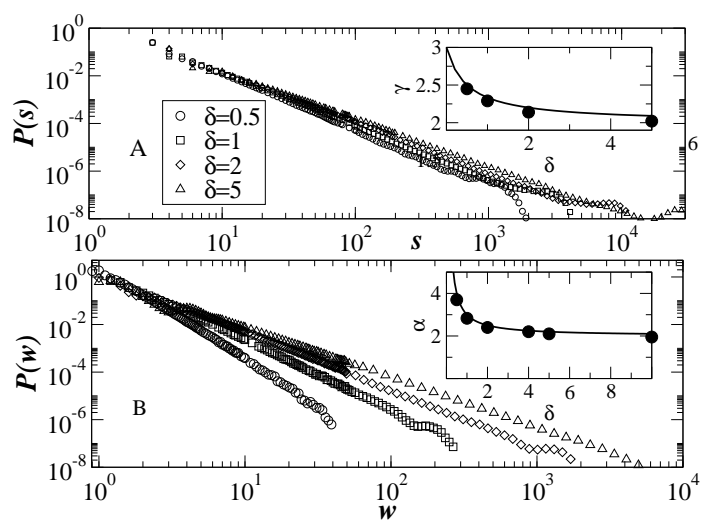

FIG. 3: (A) Probability distribution $P(s)$. Data fitting points (filled circles) are consistent with the analytic prediction of power-law behavior $s^{-\gamma}$ with $\gamma=(3+4 \delta) /(1+2 \delta)$ (solid line) as shown in the inset. (B) Probability distribution of the weights $P(w) \sim w^{-\alpha}$. In the inset we report the value of $\alpha$ obtained by data fitting (filled circles) and the analytic expression $\alpha=2+1 / \delta$ (solid line). The data are averaged over 200 networks of size $N=10^{4}$.

It is straightforward to obtain the same behavior $P(s) \sim$ $s^{-\gamma}$ for the strength distribution since $s$ and $k$ are proportional. This result shows that the obtained graph is a scale-free network described by an exponent $\gamma \in[2,3]$ that depends on the value of the parameter $\delta$. In particular, when the addition of a new edge doesn't affect the existing weights $(\delta=0)$, the model is topologically equivalent to the Barabasi-Albert model [ $[$ ] and the value $\gamma=3$ is recovered. For larger values of $\delta$ the distribution is progressively broader with $\gamma \rightarrow 2$ when $\delta \rightarrow \infty$. This indicates that the weight-driven growth generates scale-free networks with exponents varying in the range of values usually observed in the empirical analysis of networked structures 1, 3, 4]. Noticeably the exponents are non-universal and depend only on the parameter $\delta$ governing the microscopic dynamics of weights. The model therefore proposes a general mechanism for the occurrence of varying power-law behaviors without resorting on more complicate topological rules and variations of the basic preferential attachment mechanism.

In order to check the analytical predictions we performed numerical simulations of networks generated by using the present model with different value of $\delta$, minimum degree $m$ and varying size $N$. In Fig. 2] we show the behavior of the vertices' strength versus time for different value of $\delta$, recovering the behavior predicted analytically. We also report the average strength $s_{i}$ of vertices with degree $k_{i}$ and confirm that $\beta=1$ as well as the value of the pre-factor $2 \delta+1$. Finally, in Fig. 3A we plot the probability distribution $P(s)$ that exhibits a power-law behavior in excellent agreement with the theoretical predictions.

Similarly to the previous quantities, it is possible to 


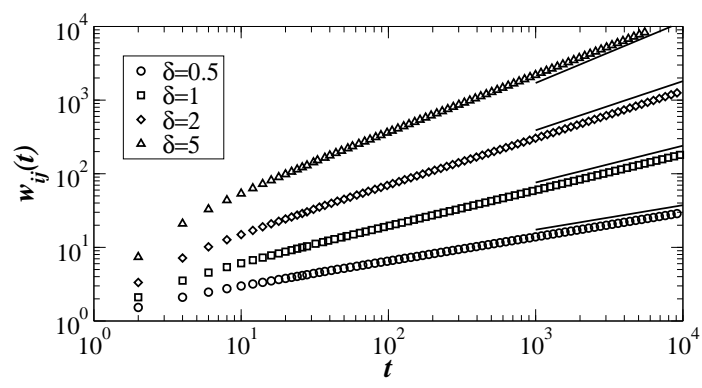

FIG. 4: Time evolution of $w_{i j}$ during the growth of the network $\left(m=2\right.$ and $\left.N=10^{4}\right)$ for different values of $\delta$. The functional behavior is consistent with the predicted power laws $t^{b}$, $b=\delta /(1+\delta)$, shown by thick lines.

obtain analytical expressions for the evolution of weights and the relative statistical distribution. The weight $w_{i j}$ increases by the addition of a new link either on $i$ or on $j$ and the corresponding rate equation can be written as

$$
\frac{d w_{i j}}{d t}=m \frac{s_{i}}{\sum_{j} s_{j}} \delta \frac{w_{i j}}{s_{i}}+m \frac{s_{j}}{\sum_{j} s_{j}} \delta \frac{w_{i j}}{s_{j}} .
$$

This equation can be solved with the initial condition $w_{i j}\left(t_{i j}\right)=1$, where $t_{i j}=\max (i, j)$ is the time at which the edge is established, yielding $w_{i j}(t)=\left(t / t_{i j}\right)^{\delta /(\delta+1)}$ From this expression it is possible to calculate similarly to $P(k)$ the probability distribution $P(w)$ which is in this case also a power-law $P(w) \sim w^{-\alpha}$ where

$$
\alpha=2+\frac{1}{\delta} .
$$

The exponent $\alpha$ has large variations as a function of the parameter $\delta$ and $P(w)$ moves from a delta function for $\delta=0$ to a very slow decaying power-law with $\alpha=2$ if $\delta \rightarrow \infty$. This feature clearly shows that the weight distribution is extremely sensitive to changes in the microscopic dynamics ruling the network's growth. These predictions can be confirmed by numerical simulations and the results are shown in Figs. $3 \mathrm{~B}$ and 4

The model we have presented is possibly the simplest one in the class of weight-driven growing networks. A novel feature in the model is the weight dynamical evolution occurring when new vertices and edges are introduced in the system. This simple mechanism produces a wide variety of complex and scale-free behavior depending on the physical parameter $\delta$ that controls the local microscopic dynamics. While a constant parameter $\delta$ is enough to produce a wealth of interesting network properties, a natural generalization of the model consists in considering $\delta$ as a function of the vertices degree or strength. For example, a non-linear generalization of eq. (4) can yield non-linear correlations between strengths and degrees $(\beta \neq 1)[19$. Similarly, more complicated variations of the microscopic rules may be implemented to mimic in a detailed fashion particular networked systems. In this perspective the present model appears as a general starting point for the realistic modeling of complex weighted networks.

We thank R. Pastor-Satorras and M. Vergassola for useful discussions. A.B and A. V. are partially funded by the EC-Fet project COSIN IST-2001-33555.

[1] R. Albert and A.-L. Barabási, Rev. Mod. Phys. 74, 47 (2000).

[2] L.A.N. Amaral, A. Scala, M. Barthélemy, and H.E. Stanley, Proc. Natl. Acad. Sci. (USA) 97, 11149 (2000).

[3] S. N. Dorogovtsev and J. F. F. Mendes, Evolution of networks: From biological nets to the Internet and $W W W$ (Oxford University Press, Oxford, 2003).

[4] R. Pastor-Satorras and A. Vespignani, Evolution and structure of the Internet: A statistical physics approach (Cambridge University Press, Cambridge, 2004).

[5] D.J. Watts and S.H. Strogatz, Nature 393, 440 (1998).

[6] A.-L. Barabási and R. Albert, Science 286, 509 (1999)

[7] S.L. Pimm, Food Webs, The University of Chicago Press (2nd edition, 2002).

[8] A.E. Krause, K.A. Frank, D.M. Mason, R.E. Ulanowicz, and W.W. Taylor, Nature 426, 282 (2003).

[9] E. Almaas, B. Kovács, T. Viscek, Z.N. Oltval and A.L. Barabási, Nature 427, 839 (2004).

[10] M. Granovetter, American Journal of Sociology, 78 (6) 1360 (1973).

[11] R. Guimera, S. Mossa, A. Turtschi, and L.A.N. Amaral, Preprint cond-mat/0312535 (2003).

[12] A. Barrat, M. Barthélemy, R. Pastor-Satorras, and A. Vespignani, Proc. Natl. Acad. Sci. USA 101, 3747 (2004).

[13] C. Li and G. Chen, cond-mat/0309236 cond-mat/0311333

[14] D. Garlaschelli, S. Battiston, M. Castri, V.D.P. Servedio, and G. Caldarelli, Preprint cond-mat/0310503 (2003).

[15] S.H. Yook, H. Jeong, A.-L. Barabasi, and Y. Tu, Phys. Rev. Lett. 86, 5835 (2001).

[16] D. Zheng, S. Trimper, B. Zheng and P.M. Hui, Phys. Rev. E 67, 040102 (2003).

[17] M. E. J. Newman, Phys. Rev. E 64, 016131 (2001); Phys. Rev. E 64, 016132 (2001).

[18] A.-L. Barabási, H. Jeong, Z. Neda, E. Ravasz, A. Schubert, and T. Vicsek, Physica A 311, 590 (2002).

[19] A. Barrat, M. Barthélemy, and A. Vespignani, in preparation (2004). 